\title{
Diagnostic Performance of F-18 Fluorocholine PET/CT for Parathyroid Localization in Hyperparathyroidism: a Systematic Review and Meta-Analysis
}

\author{
Seong-Jang Kim ${ }^{1,2,3} \cdot$ Sang-Woo Lee ${ }^{4} \cdot$ Shin Young Jeong ${ }^{4} \cdot$ Kyoungjune Pak $^{5} \cdot$ Keunyoung Kim $^{5}$
}

Received: 14 June 2018 / Accepted: 14 August 2018 / Published online: 18 August 2018

(C) Springer Science+Business Media, LLC, part of Springer Nature 2018

\begin{abstract}
The purpose of the current study was to investigate the diagnostic performance of F-18 Fluorocholine (FCH) positron emission tomography/computed tomography (PET/CT) for localization of hyperfunctioning parathyroid gland in patients with hyperparathyroidism (HPT) through a systematic review and meta-analysis. The MEDLINE/PubMed and EMBASE database, from the earliest available date of indexing through April 30, 2018, were searched for studies evaluating the diagnostic performance of F-18 FCH PET/CT for localization of hyperfunctioning parathyroid gland in patients with HPT. We determined the sensitivities and specificities across studies, calculated positive and negative likelihood ratios (LR+ and LR-), and constructed summary receiver operating characteristic curves. Across eight studies (272 patients), the pooled sensitivity for F-18 FCH PET/CT was 0.90 (95\% CI, 0.86-0.94) without heterogeneity $\left(I^{2}=7.1\right)$ and a pooled specificity of 0.94 (95\% CI, 0.90-0.96) with heterogeneity $\left(I^{2}=79.8\right)$. Likelihood ratio (LR) syntheses gave an overall positive likelihood ratio (LR+) of 5.3 (95\% CI, 1.2-24.3) and negative likelihood ratio (LR-) of 0.15 (95\% CI, 0.080.29). The pooled diagnostic odds ratio (DOR) was 38 (95\% CI, 8-174). Hierarchical summary receiver operating characteristic (ROC) curve indicates that the areas under the curve were 0.9492 (SE, 0.0215). In meta-regression analysis, no definite variable was the source of the study heterogeneity. The current meta-analysis showed the high sensitivity and specificity of F-18 FCH PET/CT for localization of hyperfunctioning parathyroid gland. At present, the literature regarding the use of F-18 FCH PET/CT for localization of hyperfunctioning parathyroid gland remains still limited; thus, further large multicenter studies would be necessary to substantiate the diagnostic accuracy of F-18 FCH PET/for localization of hyperfunctioning parathyroid gland in patients with HPT.
\end{abstract}

Keywords F-18 Fluorocholine $\cdot$ Parathyroid $\cdot$ Adenoma $\cdot$ Hyperparathyroidism

Seong-Jang Kim

growthkim@daum.net; growthkim@pusan.ac.kr

1 Department of Nuclear Medicine, Pusan National University Yangsan Hospital, Yangsan 50612, South Korea

2 BioMedical Research Institute for Convergence of Biomedical Science and Technology, Pusan National University Yangsan Hospital, Yangsan 50612, South Korea

3 Department of Nuclear Medicine, College of Medicine, Pusan National University, Yangsan 50612, South Korea

4 Department of Nuclear Medicine, Kyungpook National University Medical Center and School of Medicine, Daegu, South Korea

5 Department of Nuclear Medicine and Biomedical Research Institute, Pusan National University Hospital, Busan 49241, South Korea

\section{Introduction}

Hyperparathyroidism (HPT) is a clinical condition characterized by increased secretion of parathyroid hormone (PTH). It occurs mainly in two forms of primary hyperparathyroidism (pHPT) and secondary hyperparathyroidism (sHPT). Also, it could occur as a tertiary hyperparathyroidism mainly in chronic renal failure patients.

pHPT is the third most common endocrine disorder, with the highest incidence in postmenopausal women [1]. It is characterized by hypercalcemia associated with either elevated or nonsuppressed serum intact PTH levels. Most cases of pHPT are caused by a parathyroid adenoma and most of them are solitary lesion. Other causes include hyperplasia, double adenomas while parathyroid carcinoma is extremely infrequent $[2,3]$. 
Once the diagnosis is made, patients can be treated with either medication or surgery. However, the effective treatment is the removal of the affected parathyroid glands. Preoperative localization of the abnormal parathyroid glands could be made with various anatomical imaging modalities such as ultrasonography (US), computed tomography (CT), magnetic resonance imaging (MRI). The functional imaging techniques of Tc-99m sestamibi dual phase imaging have been used for localization of abnormal parathyroid glands [4].

Recently, F-18 Fluorocholine (FCH) positron emission tomography/computed tomography (PET/CT) was known to be able to identify pathologic parathyroid glands $[5,6]$. Choline is a part of the phospholipid layer in the cell membrane. It is hypothesized that the abnormal hyperfunctioning parathyroid gland lesions (adenoma and/or hyperplasia) revealed increased cellular proliferation, metabolism, and upregulation of choline kinase activity leading to enhanced choline uptake which allows greater imaging efficacy for the detection of abnormalities using F-18 FCH PET/CT [7]. However, not much data are available on the diagnostic performance of F-18 FCH PET/CT for localization of hyperfunctioning parathyroid gland in patients with either pHPT or sHPT. Some recent interesting studies have revealed promising and excellent results of F-18 FCH PET/ $\mathrm{CT}$ in patients with HPT even with inconclusive conventional imaging results [8-12].

The purpose of our study is to meta-analyze published data on the diagnostic accuracy of F-18 FCH PET/CT for localization of hyperfunctioning parathyroid gland, in order to provide more evidence-based data and to address further studies in the evaluation of HPT patients.

\section{Methods}

\section{Data Sources and Search Strategy}

We conducted electronic English-language literature searches of MEDLINE/PubMed and Embase database from the earliest available date of indexing through April 30, 2018. We also hand-searched the reference lists of identified publications for additional studies. We used a search algorithm based on a combination of terms (1) "PET" OR "positron emission tomography" OR "positron emission tomography/computed tomography" OR "PET/CT" OR "positron emission tomography-computed tomography" OR "PET-CT" and (2) "hyperparathyroidism" OR "parathyroid" OR "HPT" and (3) "Fluorocholine."

\section{Study Selection}

The inclusion criteria for relevant studies were as follows: F$18 \mathrm{FCH}$ PET/CT had been used to localize hyperfunctioning parathyroid gland; sufficient data to reassess sensitivity and specificity of F-18 FCH PET/CT for localization of parathyroid or absolute numbers of true positive, true negative, false positive, and false negative data had been presented; and no data overlap.

The duplicated publications were excluded, as were publications such as review articles, case reports, conference papers, and letters, which do not contain the original data. Two researchers independently reviewed titles and abstracts of the retrieved articles, applying the abovementioned selection criteria. Articles were rejected if clearly ineligible. The same researchers independently evaluated the full text of the included articles to determine their eligibility for inclusion of the current review.

\section{Data Extraction and Quality Assessment}

Information about basic study (authors, year of publication, and country of origin), study design (prospective or retrospective), patients' characteristics and technical aspects were collected.

Each study was analyzed to retrieve the number of true positive (TP), true negative (TN), false positive (FP), and false negative (FN) findings of F-18 FCH PET/CT for localization of hyperfunctioning parathyroid gland, according to the reference standard. Only studies providing such complete information were finally included in the meta-analysis.

The overall quality of the included studies in this review was critically appraised by two authors independently, based on 15-item modified Quality Assessment of Diagnostic Accuracy Studies (QUADAS2) [13]. Discrepancies between the researchers were resolved by discussion.

\section{Data Synthesis and Analysis}

All data from each eligible study were extracted. The primary objective was to estimate the sensitivity and specificity and the positive and negative likelihood ratios (LR+ and LR-, respectively) with $95 \%$ confidence intervals (CIs) and diagnostic odds ratios (DORs) with 95\% CIs. A DOR can be calculated as the ratio of the odds of positivity in a disease state relative to the odds of positivity in the non-disease state, with higher values indicating better discriminatory test performance [14]. Between-study statistical heterogeneity was assessed using $I^{2}$ and the Cochrane $Q$ test on the basis of the random-effects analysis [15]. We used the bivariate random-effects model for analysis and pooling of the diagnostic performance measures across studies, as well as comparisons between different index tests $[16,17]$. The bivariate model estimates pairs of logit transformed sensitivity and specificity from studies, incorporating the correlation that might exist between sensitivity and specificity. Each data point of the summary receiver operator characteristic (SROC) graph comes from an individual study; 
then, the SROC curve is formed based on these points to form a smooth curve to reveal pooled accuracy [18]. When statistical heterogeneity was substantial, we performed metaregression to identify potential sources of bias [19]. Twosided $p \leq 0.05$ was considered statistically significant. Statistical analyses were performed using Meta-Disc statistical software version 1.4 [20].

\section{Results}

\section{Literature Search and Selection of Studies}

After the comprehensive computerized search was performed and references lists were extensively cross-checked, our research yielded 59 records, of which 12 records of duplicated abstracts were excluded after reviewing the title and abstract. Also, non-relevant 19 studies, 2 case reports, 11 conference abstracts, and 2 review articles were excluded. Remaining 13 full-text articles were assessed for eligibility and 5 articles were excluded due to insufficient data for the calculation of sensitivity and specificity of F-18 FCH PET/CT for localization of hyperfunctioning parathyroid gland in patients with HPT. Finally, eight studies were selected and were eligible for the systematic review and meta-analysis and no additional studies were found screening the references of these articles [21-28]. The characteristics of the included studies are presented in Table 1. The detailed procedure of study selection in the current meta-analysis is shown in Fig. 1.

\section{Study Description, Quality}

We conducted all analyses based on per-lesion data. Among those eight studies included in the current review, four studies enrolled patients prospectively [21, 23, 26, 28]. Other four studies enrolled patients retrospectively $[22,24,25,27]$. There was a total of 272 patients in the included studies, and the age ranged from 22 to 87 years. A total 63 patients were male, and 192 patients were female. One study did not report the number of male and female patients in their population [27]. All eight studies analyzed the F-18 FCH PET/CT images visually. Five studies $[21-23,25,28]$ included pHPT patients and three studies $[24,26,27]$ included mixed pHPT and sHPT patients. The median value of preoperative serum calcium level was $2.68 \mathrm{mmol} / \mathrm{l}$ and ranged from 1.08 to 3.33 . Two studies did not report the preoperative serum calcium level in their studies [26, 27]. The median value of preoperative PTH level was $132 \mathrm{pg} / \mathrm{ml}$ and ranged from 15 to 1946. The principal characteristics of the eight studies included in the meta-analysis are included in Table 1.

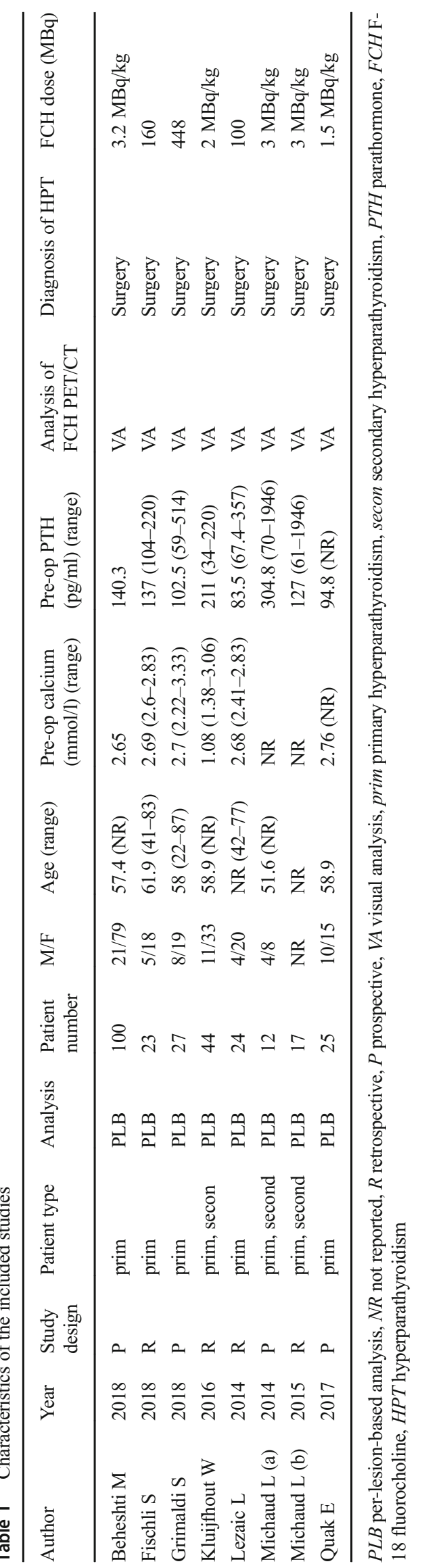




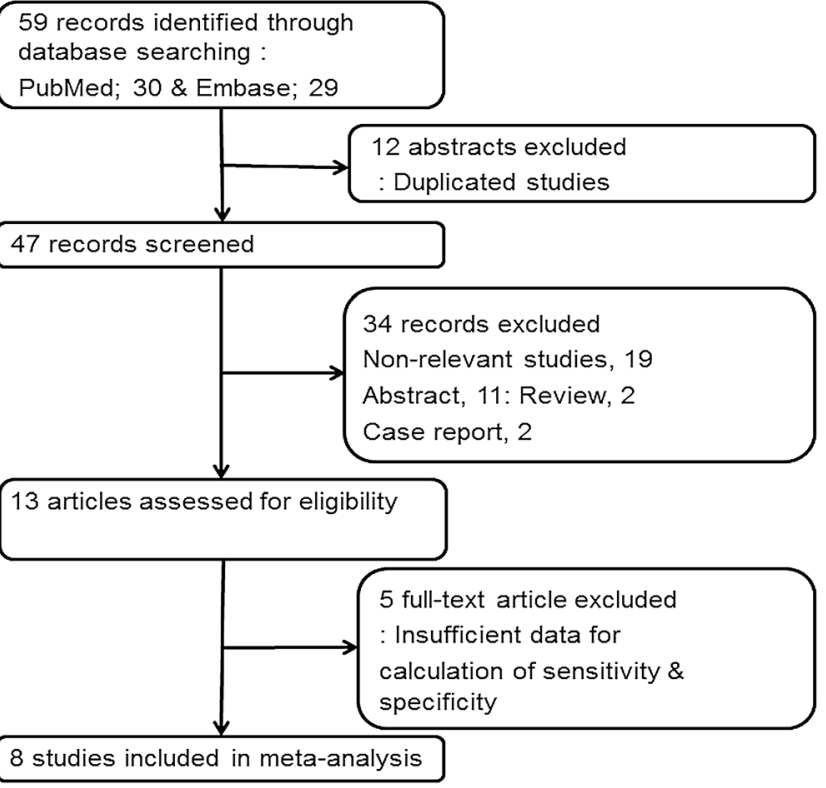

Fig. 1 Flow chart of the search for eligible studies on the diagnostic performance of F-18 FCH PET/CT for localization of hyperfunctioning parathyroid gland in patients with hyperparathyroidism (HPT)

\section{Methodological Quality Assessment}

Figure 2 shows the risk of bias and applicability concerns summary of the included studies, and overall, the quality of the included studies was deemed satisfactory.

\section{Diagnostic Accuracy of F-18 Fluorocholine PET/CT}

The diagnostic performance results of F-18 FCH PET/CT for localization of hyperfunctioning parathyroid gland in the eight included studies are presented in Fig. 3 . The pooled sensitivity

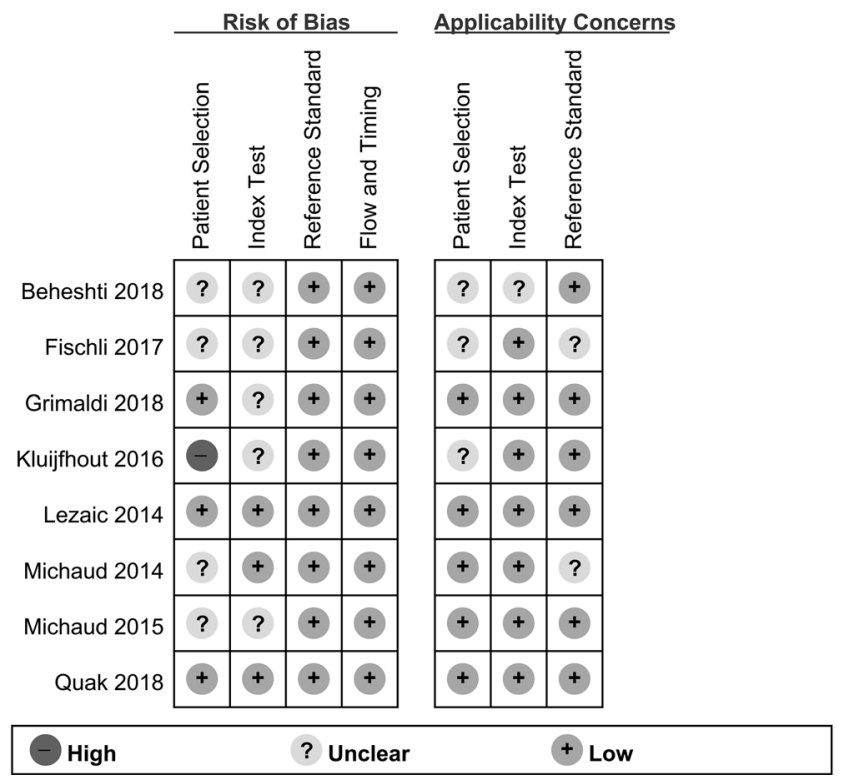

Fig. 2 Risk of bias and applicability concerns summary for F-18 FCH PET/CT was 0.90 (95\% CI, 0.86-0.94) without heterogeneity $\left(I^{2}=7.1, p=0.375\right)$ and a pooled specificity of 0.94 (95\% CI, 0.90-0.96) with heterogeneity $\left(I^{2}=79.8, p=\right.$ $0.000)$. LR syntheses gave an overall positive likelihood ratio $(\mathrm{LR}+)$ of 5.3 (95\% CI, 1.2-24.3) and negative likelihood ratio (LR-) of 0.15 (95\% CI, 0.08-0.29). The pooled DOR was 38 (95\% CI, 8-174). Forest plots of the sensitivity and specificity of F-18 FCH PET/CT are shown in Fig. 3. Figure 4 shows hierarchical summary receiver operating characteristic (ROC) curve and indicates that the area under the curve was 0.9492 (SE, 0.0215).

\section{Heterogeneity Evaluation and Meta-Regression Analysis}

Between-study heterogeneity was present for the specificity among studies of F-18 FCH PET/CT for localization of hyperfunctioning parathyroid gland. A meta-regression analysis was performed to explore other sources of heterogeneity in the studies of F-18 FCH PET/CT. Meta-regression showed that no definite variable was the source of heterogeneity in the current meta-analysis (Table 2).

\section{Discussion}

HPT is a common endocrine disease and pHPT is newly diagnosed in 100,000 patients in the USA and occurs in 0.2$0.5 \%$ of the population [29]. For the relief of the high level of PTH-related symptoms, the surgical treatment is mandatory for HPT. The previously used bilateral neck exploration is recently being replaced by minimally invasive procedures, which have the advantages of shorter duration of surgery, lower morbidity, and lower complication rates with higher success rates [30]. A prerequisite for minimally invasive procedures is accurate preoperative localization of the involved hyperfunctioning parathyroid gland. Also, this non-invasive preoperative localization of involved hyperfunctioning parathyroid gland in patients with HPT has an important impact on patient management.

Currently, the most commonly used imaging modalities for preoperative localization of involved hyperfunctioning parathyroid gland are neck US and Tc-99m methoxyisobutylisonitrile (MIBI) parathyroid scintigraphy. However, US is known to be associated with some limitations such as operator dependence, limited sensitivity for localization of ectopic parathyroid adenomas, and difficulty in differentiating adenomas from lymph nodes and thyroid nodules [31]. Tc-99m MIBI parathyroid scintigraphy is based on the abnormal accumulation in parathyroid adenoma and differential washout from thyroid and parathyroid glands. Although threedimensional imaging technique of SPECT was applied 
Fig. 3 Forest plot of pooled sensitivity and specificity of F-18 FCH PET/CT for localization of hyperfunctioning parathyroid gland in patients with HPT

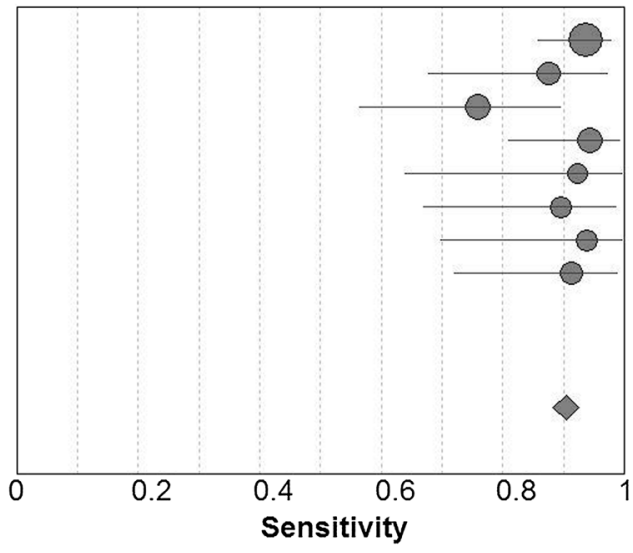

Beheshti M

Fischli S

Grimaldi S

KIujihout W

Lezaic $L$

Michaud L (2014)

Michaud L (2015)

Quak E

Sensitivity $(95 \% \mathrm{Cl})$

$0.94 \quad(0.86-0.98$

$0.88 \quad(0.68-0.97$

$0.76 \quad(0.56-0.90$

$0.94 \quad(0.81-0.99$

$0.92 \quad(0.64-1.00$

$0.89 \quad(0.67-0.99$

$0.94 \quad(0.70-1.00$

$0.91 \quad(0.72-0.99$
Pooled Sensitivity $=0.90(0.86$ to 0.94$)$

Chi-square $=7.54 ; \mathrm{df}=7(\mathrm{p}=0.3753)$

Inconsistency (I-square) $=7.1 \%$

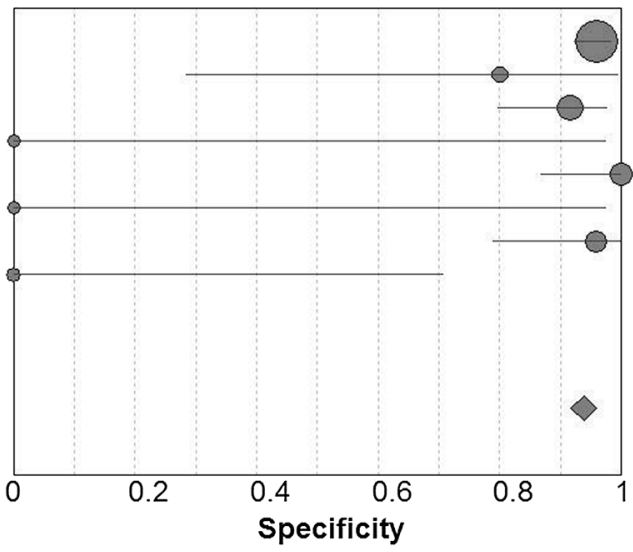

Specificity $(95 \% \mathrm{Cl})$

Beheshti M

Fischli S

Grimaldi S

Klujihout W

Lezaic $L$

Michaud L (2014)

Michaud L (2015)

Quak E

Pooled Specificity $=0.94$ (0.90 to 0.96$)$

Chi-square $=34.58 ; \mathrm{df}=7(\mathrm{p}=0.0000)$

Inconsistency (I-square) $=79.8 \%$ with Tc-99m MIBI for localization of abnormal parathyroid tissue, $25 \%$ of adenomas were false negative [32].
Recently, F-18 FCH PET/CT has been introduced as a promising imaging technique for the evaluation of in patients
Fig. 4 Hierarchical summary receiver operating characteristic (HSROC) curves of F-18 FCH $\mathrm{PET} / \mathrm{CT}$ for localization of hyperfunctioning parathyroid gland in patients with HPT

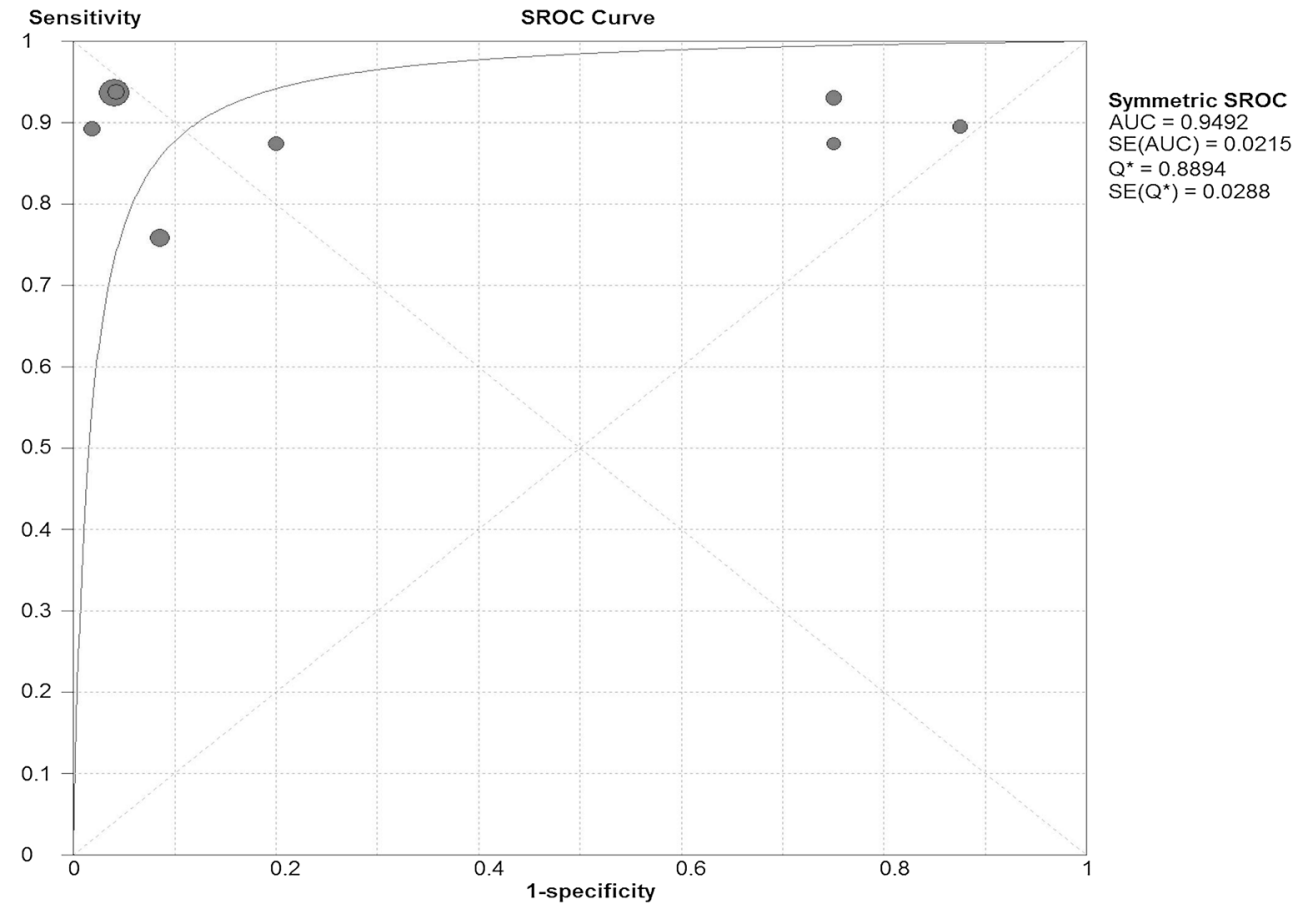


Table 2 Effects of moderators

\begin{tabular}{lllllll}
\hline Variables & Coefficient $^{\mathrm{a}}$ & SE & DOR & 95\% CI of DOR & $p^{\mathrm{b}}$ \\
\hline Number of patient $(>24$ vs $\leq 24)$ & 0.510 & 1.9013 & 1.67 & 0.00 & 5949.91 & 0.8136 \\
Study design (prospective vs retrospective) & -0.794 & 1.8604 & 0.45 & 0.00 & 1353.44 & 0.7110 \\
PTH level (> 132 vs $\leq 132 \mathrm{pg} / \mathrm{ml})$ & 0.813 & 1.4965 & 2.25 & 0.00 & 1410.16 & 0.6415 \\
Patient type (primary vs primary and & -0.672 & 1.9449 & 0.51 & 0.00 & 2200.65 & 0.7627 \\
$\quad$ secondary) & & & & & & \\
\hline
\end{tabular}

Number of patient $(1(>24)$ vs $0(\leq 24))$; study design (1 (prospective) vs 0 (petrospective)), PTH level (>132 vs $\leq 132 \mathrm{pg} / \mathrm{ml}$ ), patient type (primary vs primary and secondary)

$D O R$ diagnostic odds ratio, $S E$ standard error, $C I$ confidence interval

${ }^{a}$ Regression coefficient

${ }^{\mathrm{b}} P$ value of random effect meta-regression using maximum likelihood estimation (ML) between-study variances and the weighted least squares of study size for regression model estimation

with HPT [8-12]. Upregulation of phospholipid/ $\mathrm{Ca}^{2+}$-dependent choline kinase has been shown to be related to PTH secretion in parathyroid adenoma [7]. In a study of Beheshti et al. [21], although F-18 FCH PET-positive adenomatous lesions showed higher $\mathrm{SUV}_{\max }$ values than the hyperplastic glands in the semiquantitative analysis, the difference was not significant. Grimaldi et al. reported that F-18 FCH PET/CT is a promising modality in challenging pre-surgical localization of hyperfunctioning parathyroid glands, such as inconclusive standard imaging, recurrence after surgery, or suspected multiple gland disease [23]. In a study of Lezaic et al., the lesionbased sensitivity for F-18 FCH PET/CT was significantly better than that for Tc-99m MIBI imaging in preoperative localization of hyperfunctioning parathyroid glands [25]. Michaud et al. showed that FCH PET/CT could detect 9 adenomas and 15 hyperplastic glands [27]. According to the lesion-based analysis, the sensitivity for US was $42 \%$, whereas sensitivity for Tc-99m MIBI and F-18 FCH PET/CT was 83 and 96\%, respectively [27]. In patients with a negative or inconclusive preoperative localization with Tc-99m MIBI scintigraphy and/ or US, a detection rate of hyperfunctioning parathyroid gland by F-18 FCH PET/CT ranging between 77 and 94\% was reported on a per-patient-based analysis and between 80 and $96 \%$ on a per-lesion-based analysis $[24,28]$.

F-18 FCH PET/CT has advantages terms of better image and shorter study time owing to faster tracer kinetics and thus improved patient convenience. However, even if F-18 FCH PET/CT localization performances are quite good, FP or FN findings may occur. In a study of Grimaldi et al., they noted four FP findings related to thyroid gland uptakes and faint uptake and seven FN findings related to the ectopic localization of the parathyroid gland and multi-glandular parathyroid diseases [23].

The current meta-analysis showed a considerable heterogeneity of specificity between studies. The included studies were statistically heterogeneous in their estimates of sensitivity and specificity. This heterogeneity is likely to arise through diversity in methodological aspects between different studies and the basic differences among the patients in the included studies may have contributed to the observed heterogeneity of the results too. However, in meta-regression analysis of the current review, no definite variable was the potential source of heterogeneity. To minimize bias in the selection of studies and in the data extraction, reviewers who were blinded to the journal, author, institution, and date of publication independently selected articles based on the inclusion criteria, and scores were assigned to study design characteristics and examination results by using a standardized form that was based on the QUADAS2 tool. The results of the current meta-analysis should be interpreted with caution, and more future research is needed to confirm our results.

In addition, some major problems which could have effects on the results of the current meta-analysis should be considered. All studies included in the current meta-analysis did not report the serum level of vitamin D and creatinine. The metabolism of vitamin D is accelerated in patients with pHPT, and vitamin D deficiency is seen in up to $53 \%$ of patients with pHPT [33]. Also, sHPT occurs because of decreased levels of 1,25-dihydroxyvitamin $\mathrm{D}$, hyperphosphatemia, and hypocalcemia in chronic kidney disease. Furthermore, in patients with normocalcemic pHPT, it is important to verify normocalcemia by checking ionized calcium levels. However, not all studies reported the serum ionizing calcium level in their studies. Also, the major drawback of the current study is that all included studies did not report the exact causes of false positive and false negative cases of their studies. The administered dose of F-18 FCH in each study could affect the detection rate of parathyroid tissue localization. Therefore, when interpreting the F-18 FCH images of patients with pHPT or sHPT, cautious interpretation should be placed.

\section{Conclusion}

The current meta-analysis showed the high sensitivity and specificity of F-18 FCH PET/CT for localization of 
hyperfunctioning parathyroid gland in patients with HPT. At present, the literature regarding the use of F-18 FCH PET/CT for localization of hyperfunctioning parathyroid gland in HPT patients remains still limited; thus, further large multicenter studies would be necessary to substantiate the diagnostic accuracy of F-18 FCH PET/CT for localization of hyperfunctioning parathyroid gland in HPT patients.

Author's Contribution Protocol/project development: Kim SJ, Lee SW, Pak K

Data collection or management: Kim SJ, Kim K, Jeong SY, Pak K

Data analysis: Kim SJ, Lee SW, Kim K

Manuscript writing/editing: Kim SJ, Lee SW, Jeong SY

\section{Compliance with Ethical Standards}

Conflict of Interest The authors declare that there is no conflict of interest that could be perceived as prejudicing the impartiality of this study.

Ethical Approval Institutional review board approval was not required because we only performed data analysis based on the published studies.

Informed Consent Written informed consent was not required for this study because it is a meta-analysis based on the studies that have been published.

\section{References}

1. Fraser WD (2009) Hyperparathyroidism. Lancet 374:145-158

2. Marcocci C, Cetani F, Rubin MR, Silverberg SJ, Pinchera A, Bilezikian JP (2008) Parathyroid carcinoma. J Bone Miner Res 23:1869-1880

3. Wynne AG, van Heerden J, Carney JA, Fitzpatrick LA (1992) Parathyroid carcinoma: clinical and pathologic features in 43 patients. Medicine (Baltimore) 71:197-205

4. Taillefer R, Boucher Y, Potvin C, Lambert R (1992) Detection and localization of parathyroid adenomas in patients with hyperparathyroidism using a single radionuclide imaging procedure with technetium-99m-sestamibi (double-phase study). J Nucl Med 33: 1801-1807

5. Hodolic M, Huchet V, Balogova S, Michaud L, Kerrou K, Nataf V, Cimitan M, Fettich J, Talbot JN (2014) Incidental uptake of ${ }^{18} \mathrm{~F}$ fluorocholine $(\mathrm{FCH})$ in the head or in the neck of patients with prostate cancer. Radiol Oncol 48:228-234

6. Cazaentre T, Clivaz F, Triponez F (2014) False-positive result in ${ }^{18} \mathrm{~F}$-fluorocholine PET/CT due to incidental and ectopic parathyroid hyperplasia. Clin Nucl Med 39:e328-e330

7. Ishizuka T, Kajita K, Kamikubo K, Komaki T, Miura K, Nagao S, Nozawa Y (1987) Phospholipid/Ca2+-dependent protein kinase activity in human parathyroid adenoma. Endocrinol Jpn 34:965-968

8. Kluijfhout WP, Vorselaars WM, Vriens MR, Borel Rinkes IH, Valk GD, de Keizer B (2015) Enabling minimal invasive parathyroidectomy for patients with primary hyperparathyroidism using Tc-99msestamibi SPECT-CT, ultrasound and first results of ${ }^{18} \mathrm{~F}$ fluorocholine PET-CT. Eur J Radiol 84:1745-1751

9. Kluijfhout WP, Pasternak JD, Gosnell JE, Shen WT, Duh QY, Vriens MR, de Keizer B, Hope TA, Glastonbury CM, Pampaloni $\mathrm{MH}$, Suh I (2017) ${ }^{18} \mathrm{~F}$ Fluorocholine PET/MR imaging in patients with primary hyperparathyroidism and inconclusive conventional imaging: a prospective pilot study. Radiology 284:460-467
10. Thanseer N, Bhadada SK, Sood A, Mittal BR, Behera A, Gorla AKR, Kalathoorakathu RR, Singh P, Dahiya D, Saikia UN, Rao SD (2017) Comparative effectiveness of ultrasonography, ${ }^{99 \mathrm{~m}} \mathrm{Tc}$ sestamibi, and ${ }^{18} \mathrm{~F}$-fluorocholine $\mathrm{PET} / \mathrm{CT}$ in detecting parathyroid adenomas in patients with primary hyperparathyroidism. Clin Nucl Med 42:e491-e497

11. Taywade SK, Damle NA, Behera A, Devasenathipathy K, Bal C, Tripathi M, Agarwal S, Tandon N, Chumber S, Seenu V (2017) Comparison of ${ }^{18} \mathrm{~F}$-fluorocholine positron emission tomography/ computed tomography and four-dimensional computed tomography in the preoperative localization of parathyroid adenomasinitial results. Indian J Endocrinol Metab 21:399-403

12. Huber GF, Hüllner M, Schmid C, Brunner A, Sah B, Vetter D, Kaufmann PA, von Schulthess GK (2018) Benefit of ${ }^{18} \mathrm{~F}$ fluorocholine PET imaging in parathyroid surgery. Eur Radiol 28: 2700-2707

13. Whiting PF, Rutjes AW, Westwood ME, Mallett S, Deeks JJ, Reitsma JB, Leeflang MM, Sterne JA, Bossuyt PM, QUADAS-2 Group (2011) QUADAS-2: a revised tool for the quality assessment of diagnostic accuracy studies. Ann Intern Med 155:529-536

14. Glas AS, Lijmer JG, Prins MH, Bonsel GJ, Bossuyt PM (2003) The diagnostic odds ratio: a single indicator of test performance. J Clin Epidemiol 56:1129-1135

15. Thompson SG (1994) Why sources of heterogeneity in metaanalysis should be investigated. BMJ 309:1351-1355

16. Hamza TH, van Houwelingen HC, Stijnen T (2008) The binomial distribution of meta-analysis was preferred to model within-study variability. J Clin Epidemiol 61:41-51

17. Reitsma JB, Glas AS, Rutjes AW, Scholten RJ, Bossuyt PM, Zwinderman AH (2005) Bivariate analysis of sensitivity and specificity produces informative summary measures in diagnostic reviews. J Clin Epidemiol 58:982-990

18. Rutter CM, Gatsonis CA (2001) A hierarchical regression approach to meta-analysis of diagnostic test accuracy evaluations. Stat Med 20:2865-2884

19. Lijmer JG, Mol BW, Heisterkamp S, Bonsel GJ, Prins MH, van der Meulen JH, Bossuyt PM (1999) Empirical evidence of designrelated bias in studies of diagnostic tests. JAMA 282:1061-1066

20. Zamora J, Abraira V, Muriel A, Khan K, Coomarasamy A (2006) Meta-DiSc: a software for meta-analysis of test accuracy data. BMC Med Res Methodol 6:31

21. Beheshti M, Hehenwarter L, Paymani Z, Rendl G, Imamovic L, Rettenbacher R, Tsybrovskyy O, Langsteger W, Pirich C (2018) ${ }^{18} \mathrm{~F}$-Fluorocholine $\mathrm{PET} / \mathrm{CT}$ in the assessment of primary hyperparathyroidism compared with ${ }^{99 \mathrm{~m}} \mathrm{Tc}-\mathrm{MIBI}$ or $99 \mathrm{~m} \mathrm{Tc}$-tetrofosmin SPECT/CT: a prospective dual-centre study in 100 patients. Eur J Nucl Med Mol Imaging 45:1762-1771. https://doi.org/10.1007/ s00259-018-3980-9

22. Fischli S, Suter-Widmer I, Nguyen BT, Müller W, Metzger J, Strobel K, Grünig H, Henzen C (2018) The significance of ${ }^{18} \mathrm{~F}$ fluorocholine-PET/CT as localizing imaging technique in patients with primary hyperparathyroidism and negative conventional imaging. Front Endocrinol (Lausanne) 8:380

23. Grimaldi S, Young J, Kamenicky P, Hartl D, Terroir M, Leboulleux S, Berdelou A, Hadoux J, Hescot S, Remy H, Baudin E, Schlumberger M, Deandreis D (2018) Challenging pre-surgical localization of hyperfunctioning parathyroid glands in primary hyperparathyroidism: the added value of ${ }^{18} \mathrm{~F}$ fluorocholine PET/CT. Eur J Nucl Med Mol Imaging. https:// doi.org/10.1007/s00259-018-4018-z

24. Kluijfhout WP, Vorselaars WM, van den Berk SA, Vriens MR, Borel Rinkes IH, Valk GD, van Dalen T, de Klerk JM, de Keizer B (2016) Fluorine-18 fluorocholine PET-CT localizes hyperparathyroidism in patients with inconclusive conventional imaging: a multicenter study from the Netherlands. Nucl Med Commun 37:1246-1252 
25. Lezaic L, Rep S, Sever MJ, Kocjan T, Hocevar M, Fettich J (2014) ${ }^{18}$ F-Fluorocholine PET/CT for localization of hyperfunctioning parathyroid tissue in primary hyperparathyroidism: a pilot study. Eur J Nucl Med Mol Imaging 41:2083-2089

26. Michaud L, Burgess A, Huchet V, Lefèvre M, Tassart M, Ohnona J, Kerrou K, Balogova S, Talbot JN, Périé S (2014) Is ${ }^{18} \mathrm{~F}$ fluorocholine-positron emission tomography/computerized tomography a new imaging tool for detecting hyperfunctioning parathyroid glands in primary or secondary hyperparathyroidism? J Clin Endocrinol Metab 99:4531-4536

27. Michaud L, Balogova S, Burgess A, Ohnona J, Huchet V, Kerrou K, Lefèvre M, Tassart M, Montravers F, Périé S, Talbot JN (2015) A pilot comparison of ${ }^{18} \mathrm{~F}$-fluorocholine PET/CT, ultrasonography and ${ }^{123} \mathrm{I} /{ }^{99 \mathrm{~m}} \mathrm{Tc}$-sestaMIBI dual-phase dual-isotope scintigraphy in the preoperative localization of hyperfunctioning parathyroid glands in primary or secondary hyperparathyroidism: influence of thyroid anomalies. Medicine (Baltimore) 94:e1701

28. Quak E, Blanchard D, Houdu B, Le Roux Y, Ciappuccini R, Lireux B, de Raucourt D, Grellard JM, Licaj I, Bardet S, Reznik Y, Clarisse B, Aide N (2018) F18-choline PET/CT guided surgery in primary hyperparathyroidism when ultrasound and MIBI SPECT/CT are negative or inconclusive: the APACH1 study. Eur J Nucl Med Mol Imaging 45:658-666

29. Allendorf J, Kim L, Chabot J, DiGiorgi M, Spanknebel K, LoGerfo P (2003) The impact of sestamibi scanning on the outcome of parathyroid surgery. J Clin Endocrinol Metab 88:3015-3018

30. Lew JI, Solorzano CC (2009) Surgical management of primary hyperparathyroidism: state of the art. Surg Clin N Am 89: 1205-1225

31. Johnson NA, Tublin ME, Ogilvie JB (2007) Parathyroid imaging: technique and role in the preoperative evaluation of primary hyperparathyroidism. AJR Am J Roentgenol 188:1706-1715

32. Bergenfelz AO, Jansson SK, Wallin GK, Mårtensson HG, Rasmussen L, Eriksson HL, Reihnér EI (2009) Impact of modern techniques on short-term outcome after surgery for primary hyperparathyroidism: a multicenter study comprising 2,708 patients. Langenbeck's Arch Surg 394:851-860

33. Perrier ND (2007) Impact of 25 hydroxyvitamin D deficiency in perioperative parathyroid hormone kinetics and results in patients with primary hyperparathyroidism. Surgery 142:1027-1029 